\title{
Microencapsulation Method for Atom Probe Analysis of Powders
}

\author{
David C. Bell ${ }^{1}$, Andrew P. Magyar², Adam C. Graham² and Mor Baram ${ }^{1}$ \\ 1. School of Engineering and Applied Sciences, Harvard University, Cambridge MA USA \\ 2. Center for Nanoscale Systems, Harvard University, Cambridge MA USA
}

Recent interest in nano-particles for applications in alternative energy technologies such as low temperature solid-oxide fuel cells, catalysts, and battery electrodes has created a need for controlled microstructures and the examination of powders and materials both chemically and structurally at the atomic level. 3D Local Electrode Atom Probe Tomography (LEAP) is the only tool available that can provide information about both composition and distribution with sufficient spatial resolution for these applications.[1] However, the nature of these typically porous samples has made analysis by Atom Probe Tomography (APT) or LEAP seriously demanding due to sample fracture and other factors.

We have developed a novel glass micro-encapsulation technique to examine nanoparticles or micronsized powder samples using LEAP. Successful formation of a specimen for LEAP analysis is heavily dependent on the specifics of sample preparation. A glass fiber micro capillary tube is firstly filled with the powder or nanoparticles to be examined, then gently heated and extended with our constant force fiber extractor (Fig.1). The resultant sample is a micro-fiber with a nanoscale radius of curvature of the tip. To mount the sample for LEAP analysis the capillary tip was cleaved to produce a segment approximately $2 \mathrm{~mm}$ in length. This segment was laminated to metal post using cyanoacrylate adhesive (Super Glue) (Fig. 2).

If required, the capillary tip can be further shaped by FIB to provide the ideal tip geometry for LEAP characterization, however through fastidious control of the initial tip formation such post-processing is not necessary. Commercially available micropipette pullers, typically used to produce optical fiber probes and nanospray tips, can provide even greater parametric control over the tip formation enabling tips with radii as small as $50 \mathrm{~nm}$ [2]. Alternatively, after initial capillary drawing, wet etching in HF can be employed to shape tips. [2]

The technique we demonstrate for the encapsulation of particulate materials within an atom probe tip provides a rapid, robust, and inexpensive way to analyze nano- or micro-particles using LEAP (Fig. 3). Employing this method with industrially relevant materials such as catalysts can provide insights into the composition at the nanostructural level and consequently provide a better understanding of factors driving efficiency and failure in such materials.

[1] Gault, Moody, Cairney and Ringer, Atom Probe Microscopy, Springer 2012

[2] Hoffmann, Dutoit, and Salathé, "Comparison of mechanically drawn and protection layer chemically etched optical fiber tips," Ultramicroscopy 61, 165-170 (1995). 

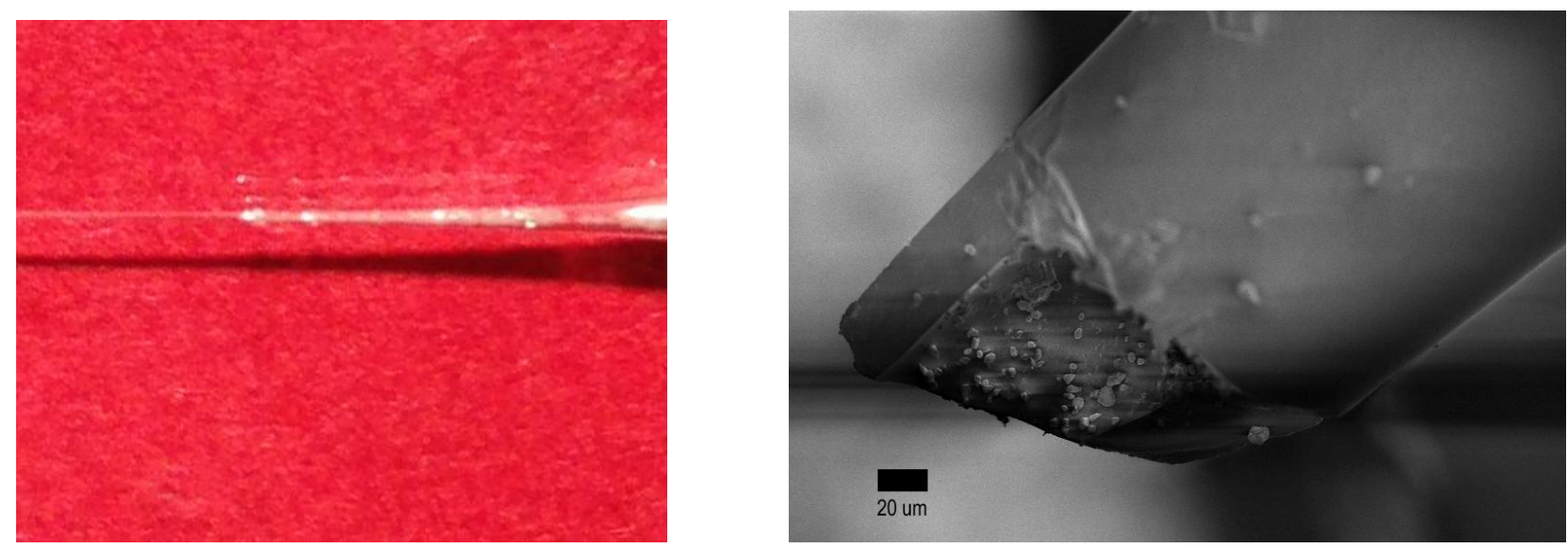

Figure 1. Left, pulled glass fiber capillary showing the micro-powder clearly at the thicker end. Right, pulled glass fiber capillary (broken) showing the micro/nano particle distribution.

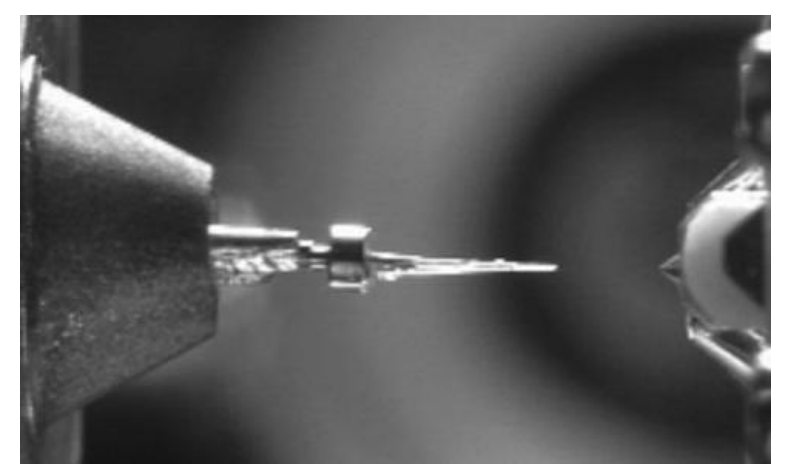

Figure 2. Image of nano-tapered silica capillary in the atom probe microscope.

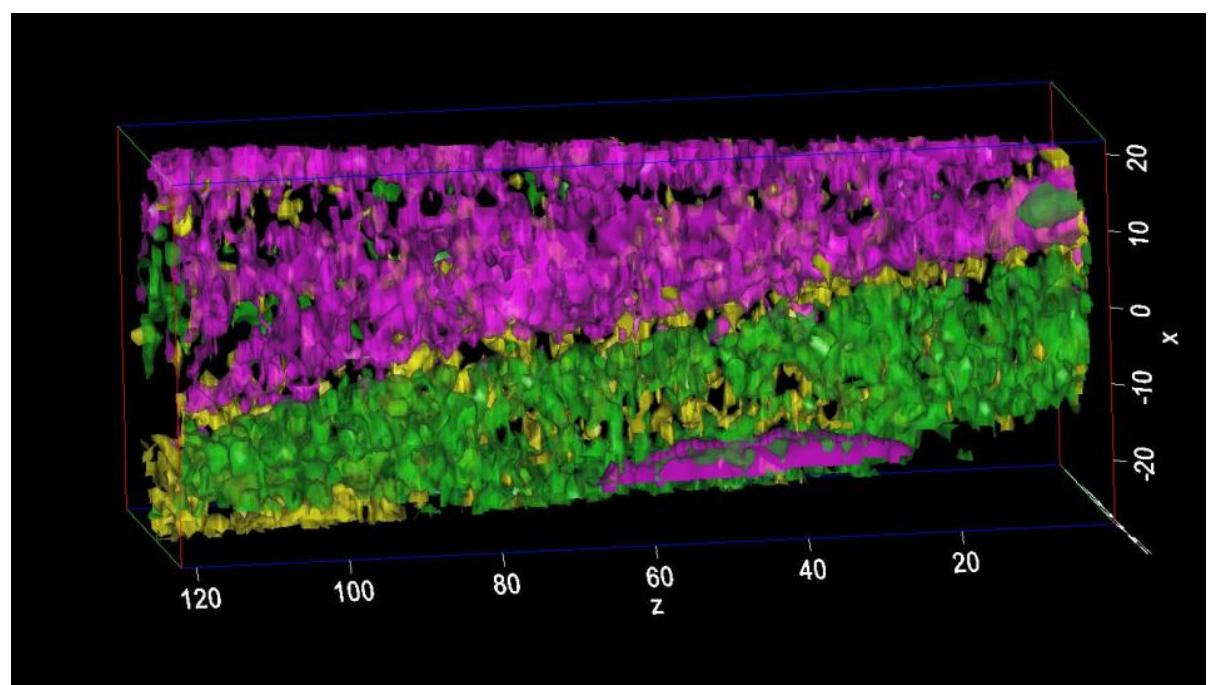

Figure 3. Steel/Ni/Cr nanoparticle matrix sample, showing nanoparticle distribution as iso-surface concentrations. 